\title{
Galois actions on regular dessins of small genera
}

\author{
Marston D. E. Conder, Gareth A. Jones, Manfred Streit \\ and Jürgen Wolfart
}

\begin{abstract}
Dessins d'enfants can be regarded as bipartite graphs embedded in compact orientable surfaces. According to Grothendieck and others, a dessin uniquely determines a complex structure on the surface, and even an algebraic structure (as a projective algebraic curve defined over a number field). The general problem of how to determine all properties of the curve from the combinatorics of the dessin is far from being solved. For regular dessins, which are those having an edge-transitive automorphism group, the situation is easier: currently available methods in combinatorial and computational group theory allow the determination of the fields of definition for all curves with regular dessins of genus 2 to 18 .
\end{abstract}

\section{The many facets of dessins}

One may introduce dessins d'enfants as hypermaps on compact oriented 2-manifolds. These objects, first studied in genus 0 by Cori in [9], have several equivalent algebraic or topological definitions. Topologically they are a generalisation of maps, whose underlying graphs are replaced with hypergraphs, in which hyperedges are allowed to be incident with any finite number of hypervertices and hyperfaces. One way to visualise this concept is to represent a hypermap $\mathcal{D}$ by its Walsh map $W(\mathcal{D})$, a connected bipartite graph $\mathcal{B}$ embedded in a compact oriented surface, dividing it into simply connected cells [31]. In the language of hypermaps, the white and black vertices represent the hypervertices and hyperedges of $\mathcal{D}$, the edges represent incidences between them, and the cells represent the hyperfaces.

There are several other ways to define dessins. There is a group theoretic description of maps and hypermaps by their (hyper)cartographic groups; see, for example, [18], [19] or the introduction of [20]. The latter are the monodromy groups of the functions about to be introduced, and give a link to function theory on Riemann surfaces and algebraic geometry on curves. Playing a key role are

Mathematics Subject Classification (2010): Primary 14H45; Secondary 14H25, 14H30, 14H37, 14H55, 11G32, 05C10, 05C25, 30F10.

Keywords: Regular maps and hypermaps, dessins d'enfants, Galois actions, fields of definition. 
Belyॅ functions, which are non-constant meromorphic functions

$$
\beta: X \rightarrow \mathbb{P}^{1}(\mathbb{C})
$$

on a Riemann surface $X$, ramified above at most three points, customarily normalised to be 0,1 and $\infty$. According to Belyı's theorem [2], such functions exist if and only if $X$ can be defined (as a smooth projective algebraic curve) over a number field.

Two observations by Grothendieck [14] are crucial. The first is that to every Bely $\breve{1}$ function belongs a dessin. In the Walsh representation, we can see the zeros of $\beta$ as white vertices, the points in $\beta^{-1}(1)$ as black vertices, and the connected components of the $\beta$-preimage of the real interval $(0,1)$ as the edges of the bipartite graph; in this picture, the poles of $\beta$ play the role of the cell midpoints. Grothendieck stressed a second point whose proof can be traced back to [24] and [17] (some years before Belyı̌'s discovery), namely that every dessin $\mathcal{D}$ defines a unique conformal structure on $X$ and a Belyu function on $X$ inducing $\mathcal{D}$, and hence even a structure as an algebraic curve over $\overline{\mathbb{Q}}$. As a consequence, one has a natural (indeed faithful) action of the absolute Galois group Gal $\overline{\mathbb{Q}} / \mathbb{Q}$ on the set of all dessins, induced by the action on the curves and the Belyu functions - or more precisely, on the equation coefficients of the curves and the coefficients of the Belyı functions. In particular, the fields of definition of the curves and their Belyı functions should be encoded in their dessins.

These will form the main topic of this paper, but first we need to introduce another tool, namely the link between dessins and triangle groups.

On Riemann surfaces $X$ that are uniformised by subgroups $\Gamma$ of finite index in a triangle group $\Delta$, there exist natural Bely functions as covering maps

$$
\beta: X=\Gamma \backslash \mathbb{H} \rightarrow \Delta \backslash \mathbb{H}
$$

where $\mathbb{H}$ is the hyperbolic plane if $\Delta$ is Fuchsian (while in the few cases where $\Delta$ is a spherical or euclidean triangle group, $\mathbb{H}$ is the Riemann sphere $\mathbb{P}^{1}(\mathbb{C})$ or the Euclidean plane $\mathbb{C}$, respectively). Conversely, every Belyı̆ function can be realised in this way; in particular, every Belyı surface $X$ (that is, every Riemann surface endowed with a Belyı̆ function) can be described as the quotient space $\Gamma \backslash \mathbb{H}$ for some subgroup $\Gamma$ of a Fuchsian triangle group $\Delta$; see [4].

In this context, the bipartite graph of the Walsh map is part of the skeleton induced by the tessellation of $\mathbb{H}$ obtained from the usual fundamental domain for $\Delta$. Figure 1 shows a typical example of this construction; the left hand side is due to Felix Klein [22], the right hand side gives the dessin belonging to this tessellation, and the numbers on the boundary of the fundamental domain of $\Gamma$ indicate the identifications needed to obtain the surface.

The signature $\langle p, q, r\rangle$ of the triangle group $\Delta$ is related to the Belyu function $\beta$ as follows: $p$ is a common multiple of the zero orders of $\beta$, while $q$ is a common multiple of the zero orders of $\beta-1$, and $r$ is a common multiple of the pole orders of $\beta$ (which in turn are visible via the valencies of the dessin).

Finally, we wish to explain that we restrict our attention in this paper to regular dessins, namely those dessins having an edge-transitive, colour- and orientationpreserving automorphism group. In the literature, these are also known as "rotary" 

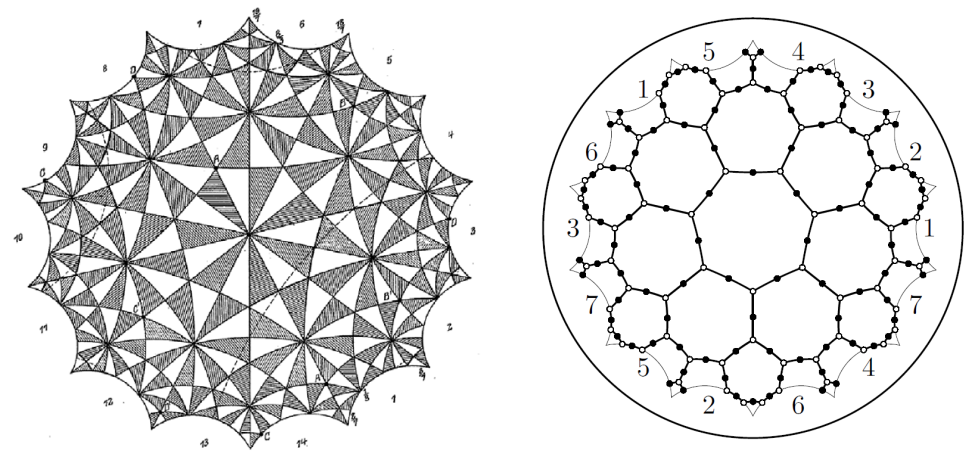

Figure 1. Tessellation and dessin for Klein's quartic $x^{3} y+y^{3} z+z^{3} x=0$.

or "orientably regular" hypermaps. Note that the automorphism group also acts as an automorphism group of the underlying Riemann surface. In the Walsh map of a regular dessin, all white vertices have the same valency, as do all black vertices, and all cells (hyperfaces) have the same size. Hence in the uniformisation context above, the subgroup $\Gamma$ is a torsion-free normal subgroup of the triangle group $\Delta$. The quotient $\Delta / \Gamma$ is isomorphic to the automorphism group of the dessin, and to the covering group of $\beta$; in this case it is a normal ramified cover $X \rightarrow \mathbb{P}^{1}(\mathbb{C})$. Because of its $\Delta$-invariant triangular tessellation, the surface $X$ is called quasiplatonic.

Regular dessins and quasiplatonic surfaces have many other interesting properties; see for example Theorem 4 in [35], or [27]. There are only finitely many regular dessins of any given genus $g>1$, and they are all known for genera 2 to 100 (see [6]). Every quasiplatonic surface $X$ of genus $g>1$ has automorphism group Aut $X \cong \Delta / \Gamma$ for some triangle group $\Delta$, uniquely determined as the normaliser of the universal covering group $\Gamma$ in $\mathrm{PSL}_{2} \mathbb{R}$, and defining a maximal regular dessin on $X$. (Other regular dessins may exist on $X$, coming from intermediate triangle subgroups between $\Delta$ and $\Gamma$; see [25].) What is important for this paper is our experience that the fields of definition for regular dessins and quasiplatonic surfaces are easier to determine than those of arbitrary dessins or Belyı̆ surfaces. Moreover, they play a systematic role for all dessins, because every dessin is obtainable as a quotient of some regular dessin by a subgroup of its automorphism group.

\section{Known methods and their variants}

In only a few cases, such as quasiplatonic curves of genus up to 4 [33], or for special families, such as the Fermat curves, are explicit equations known for the curves, or are explicit representations for the Belyı̆ functions known as rational functions on these curves. Generally it is difficult to determine a minimal field (or even a small field) of definition for the curve or the dessin - that is, for both the curve $X$ and the corresponding Belyı function $\beta$. It is easier to determine instead the field of moduli of the curve $X$, defined as the fixed field of all $\sigma \in \mathrm{Gal} \overline{\mathbb{Q}} / \mathbb{Q}$ for which 
there is an isomorphism $f_{\sigma}: X \rightarrow X^{\sigma}$. For a dessin, this isomorphism $f_{\sigma}$ has to be compatible with the Belyu function $\beta$ as well. Clearly the field of moduli is contained in every field of definition, and depends only on the isomorphism class of the curve or the dessin. Here, an isomorphism between two dessins $\left(X_{1}, \beta_{1}\right)$ and $\left(X_{2}, \beta_{2}\right)$ is an isomorphism of curves $f: X_{1} \rightarrow X_{2}$ compatible with the respective Belyŭ functions (that is, such that $\beta_{2} \circ f=\beta_{1}$ ), and hence is also a graph isomorphism.

By ideas from [8] (see also [10] and Theorem 5 in [35]), we have

Proposition 1. The fields of moduli of regular dessins and of quasiplatonic curves are their respective minimal fields of definition.

As another tool, we can use Galois invariants of dessins, many of which were given in [19]. We call the signature $\langle p, q, r\rangle$ of the triangle group $\Delta$ also the signature of the dessin, if the parameters $p, q, r$ are chosen minimally. We easily obtain the following:

Proposition 2. The genus, the signature, and the isomorphism class of the automorphism group are Galois invariants of a dessin.

From the two propositions above, we can draw an easy conclusion which is applicable to many regular dessins and quasiplatonic curves, especially those of small genus.

Theorem 1. If a regular dessin is uniquely determined up to isomorphism by its genus, signature and automorphism group, then it can be defined over the field $\mathbb{Q}$ of rational numbers. Accordingly, a quasiplatonic curve can be defined over $\mathbb{Q}$ if it has a regular dessin with this property.

The latter applies to all quasiplatonic curves and their maximal dessins with genus $g$ lying in the range $1<g<6$, as can be verified using the classification [6] that this paper is based on. In genus 6 , however, we find there are non-isomorphic dessins with common signature $\langle 7,14,14\rangle$ and common automorphism group $C_{14}$ (cyclic of order 14). They give the first candidates for a nontrivial Galois action on quasiplatonic curves, but the fields of definition turn out to be $\mathbb{Q}$ for all these dessins:

Proposition 3. If the automorphism group of a regular dessin is abelian, then the dessin (and its underlying quasiplatonic curve) can be defined over $\mathbb{Q}$.

This may be seen as a special case of a more general theorem concerning socalled homology covers, which allows the explicit determination of defining equations for the curve [15]. We are grateful to Benjamin Mühlbauer for an alternative argument from his forthcoming $\mathrm{PhD}$ thesis, which uses the fact that every such dessin is a quotient of a regular dessin of signature $\langle n, n, n\rangle$ living on the Fermat curve $F_{n}$ of exponent $n$ with automorphism group $C_{n} \times C_{n}$. A closer look at the action of this automorphism group on $F_{n}$ shows that every subgroup is Galois invariant, and therefore so is any quotient dessin. Hence all such quotients have moduli field $\mathbb{Q}$, and the result follows from Proposition 1. 
To handle nontrivial Galois actions, an observation made recently in [20] is extremely useful: Galois actions on hypermaps are often equivalent to Wilson operators [32]. With regard to the triangle group description of a dessin, these Wilson operators $H_{j}$ can be defined as follows.

Suppose $\Delta$ has signature $\langle p, q, r\rangle$, so that $\Delta$ has a presentation

$$
\left\langle\gamma_{0}, \gamma_{1} \mid \gamma_{0}^{p}=\gamma_{1}^{q}=\left(\gamma_{0} \gamma_{1}\right)^{r}=1\right\rangle
$$

in terms of hyperbolic rotations $\gamma_{0}$ and $\gamma_{1}$ with rotation angles $\frac{2 \pi}{p}$ and $\frac{2 \pi}{q}$ at neighbouring fixed points (which project onto neighbouring white and black vertices of the dessin). Then the universal covering group $\Gamma$ of the underlying quasiplatonic surface is the kernel of an epimorphism

$$
h: \Delta \rightarrow G \cong \text { Aut } \mathcal{D} \text { taking }\left(\gamma_{0}, \gamma_{1}\right) \mapsto(a, b)
$$

for some $a, b \in G$, such that $a, b$ and $a b$ have orders $p, q$ and $r$ respectively.

Now for any unit $j$ in the ring $\mathbb{Z}_{p q}=\mathbb{Z} / p q \mathbb{Z}$, let $r^{\prime}$ be the order of $a^{j} b^{j}$, and let $\Delta^{\prime}$ be the triangle group with signature $\left\langle p, q, r^{\prime}\right\rangle$. Then since $a^{j}$ and $b^{j}$ have orders $p$ and $q$ respectively, there exists an epimorphism $h_{j}: \Delta^{\prime} \rightarrow G$ taking $\left(\gamma_{0}, \gamma_{1}\right) \mapsto\left(a^{j}, b^{j}\right)$, with torsion-free kernel $\Gamma_{j}$. This gives a regular $\operatorname{dessin} H_{j} \mathcal{D}$ on $X_{j}:=\Gamma_{j} \backslash \mathbb{H}$, with automorphism group again isomorphic to $G$.

We may consider the dessin $H_{j} \mathcal{D}$ as the result of applying the Wilson operator $H_{j}$ to the regular dessin $\mathcal{D}$. Geometrically, $H_{j} \mathcal{D}$ is a regular embedding of the original graph into a possibly different quasiplatonic surface, obtained by an obvious change of the local cyclic ordering of the edges around the vertices. Note that this may give new faces (if $j \neq 1$ ), and can even give a different genus (if $\left.r^{\prime} \neq r\right)$. The genus is preserved in the special case of the Wilson operator $H_{-1}$, which takes every such $\mathcal{D}$ to its mirror image, and therefore transposes every chiral pair of regular dessins, that is a pair of non-isomorphic dessins interchanged under an anticonformal homeomorphism of the surface.

There are also generalisations $H_{i, j}$ of these Wilson operators to situations where $a$ and $b$ are replaced with $a^{i}$ and $b^{j}$ for units $i$ and $j$ in $\mathbb{Z}_{p}$ and $\mathbb{Z}_{q}$ respectively; see $[20]$.

By Theorems 2 and 3 of [20], we have the following:

Proposition 4. Let $\left\{\mathcal{D}_{j} \mid j \in(\mathbb{Z} / m \mathbb{Z})^{*}\right\}$ be a family of regular dessins $\mathcal{D}_{j}=$ $\left(X_{j}, \beta_{j}\right)$, each having signature $\langle p, q, r\rangle$, such that $m$ is the least common multiple of $p$ and $q$. Suppose that these regular dessins form a single orbit under Wilson's map operators $H_{j}$, with $\mathcal{D}_{j}=H_{j} \mathcal{D}_{1}$ for all $j$, and also that this family is invariant under the action of the absolute Galois group $\mathrm{Gal} \overline{\mathbb{Q}} / \mathbb{Q}$. Then the curves $X_{j}$ (as smooth projective algebraic curves) and their Belyu functions $\beta_{j}$ can be defined over a subfield $K$ of the cyclotomic field $\mathbb{Q}\left(\zeta_{m}\right)$, where $\zeta_{m}=e^{2 \pi i / m}$, and the given family forms a single orbit under the action of the absolute Galois group. Here the subfield $K$ is the fixed field of the subgroup

$$
H:=\left\{j \in(\mathbb{Z} / m \mathbb{Z})^{*} \mid H_{j} \mathcal{D}_{1} \cong \mathcal{D}_{1}\right\},
$$

when we identify $(\mathbb{Z} / m \mathbb{Z})^{*}$ with the Galois group $\operatorname{Gal} \mathbb{Q}\left(\zeta_{m}\right) / \mathbb{Q}$. 
Proposition 5. Let $\left\{\mathcal{D}_{i, j} \mid(i, j) \in S\right\}$ be a family of regular dessins $\mathcal{D}_{i, j}=$ $\left(X_{i, j}, \beta_{i, j}\right)$, each having signature $\langle p, q, r\rangle$ and indexed by a subset $S$ of $(\mathbb{Z} / p \mathbb{Z})^{*} \times$ $(\mathbb{Z} / q \mathbb{Z})^{*}$ admitting an action of $(\mathbb{Z} / m \mathbb{Z})^{*}$ where $m$ is the least common multiple of $p$ and $q$. (This means that whenever $S$ contains $(i, j)$, it also contains $(k i, k j)$ for all $k \in(\mathbb{Z} / m \mathbb{Z})^{*}$.) Suppose that these regular dessins form a single orbit under the Wilson map operators $H_{i, j}$, with $\mathcal{D}_{i, j}=H_{i, j} \mathcal{D}_{1,1}$ for all $i, j$, and also that this family is invariant under the action of the absolute Galois group $\mathrm{Gal} \overline{\mathbb{Q}} / \mathbb{Q}$.

Then the curves $X_{i, j}$ (as smooth projective algebraic curves) and their Belyı functions $\beta_{i, j}$ can be defined over a subfield $K$ of the cyclotomic field $\mathbb{Q}\left(\zeta_{m}\right)$, where $\zeta_{m}=e^{2 \pi i / m}$. Furthermore, the orbits of the action of the absolute Galois group are precisely the orbits of the action of the set of Wilson operators $\left\{H_{k} \mid k \in(\mathbb{Z} / m \mathbb{Z})^{*}\right\}$ on this family - that is, the orbit of $\mathcal{D}_{i, j}$ consists of

$$
H_{k i, k j} \mathcal{D}_{1,1}=H_{k} H_{i, j} \mathcal{D}_{1,1}=H_{k} \mathcal{D}_{i, j} \quad \text { for all } k \in(\mathbb{Z} / m \mathbb{Z})^{*} .
$$

Also the minimal field of definition of each $\mathcal{D}_{i, j}=\left(X_{i, j}, \beta_{i, j}\right)$ is the fixed field $K \subseteq \mathbb{Q}\left(\zeta_{m}\right)$ of the subgroup of all $k \in(\mathbb{Z} / m \mathbb{Z})^{*}$ for which $\mathcal{D}_{i, j} \cong \mathcal{D}_{k i, k j}$.

It can often happen that not all Wilson operators produce Galois conjugate curves - for example, if $H_{j} \mathcal{D}$ is a dessin on a curve of different genus from that of $\mathcal{D}$. In some of these cases, precisely the same arguments as in the proof of Theorem 2 in [20] give the following slight generalisation.

Theorem 2. Under the same hypotheses as in Proposition 4, but with $j$ running over a proper subgroup $U$ of $(\mathbb{Z} / m \mathbb{Z})^{*}$, the curves $X_{j}$ and their Belyı functions $\beta_{j}$ can be defined over a subfield $K$ of the cyclotomic field $\mathbb{Q}\left(\zeta_{m}\right)$, and the Belyı pairs $\left(X_{j}, \beta_{j}\right)$ form a single orbit under the action of the absolute Galois group. If we consider $U$ in the usual way as a subgroup of $\mathrm{Gal} \mathbb{Q}\left(\zeta_{m}\right) / \mathbb{Q}$, then $U$ acts by restriction as the Galois group $\mathrm{Gal} K / \mathbb{Q}$; in particular, the index $|U: H|$ of the subgroup $H:=\left\{j \in U \mid H_{j} \mathcal{D}_{1} \cong \mathcal{D}_{1}\right\}$ is equal to the degree $[K: \mathbb{Q}]$ of $K$ as an extension of $\mathbb{Q}$.

For example, if $U=\{ \pm 1\}$ and $H_{-1} \mathcal{D} \neq \mathcal{D}$, then we know that the regular dessins $\mathcal{D}$ and $H_{-1} \mathcal{D}$ form a chiral pair defined over an imaginary quadratic subfield of $\mathbb{Q}\left(\zeta_{m}\right)$ - which is often uniquely determined. Proposition 5 can be generalised in a similar manner, of course.

Galois actions on families of quasiplatonic curves cannot always be described by Wilson operators, as shown in Corollary A1 of [20]. In cases where the automorphism group is $\mathrm{PSL}_{2} \mathbb{F}_{q}$ or an extension of this, and in particular if $\Delta$ is arithmetically defined, then the methods developed in [26], [11] and [12] are more applicable. We quote a special case (as an example), explaining Galois actions on many Hurwitz curves.

Proposition 6. Let $\Delta$ be the triangle group of signature $\langle 2,3,7\rangle$. The pre-image of $\Delta$ in $\mathrm{SL}_{2} \mathbb{R}$ is the norm 1 group of a maximal order in a quaternion algebra defined over the cubic field $k:=\mathbb{Q}(\cos 2 \pi / 7)$. Let $p$ be a rational prime, and let $\wp$ be a prime ideal in the ring $\mathcal{O}$ of integers of $k$, lying over $p$ and with norm 
$N(\wp)=q=p^{f}$. Then the principal congruence subgroup $\Delta(\wp)$ is a surface group for some surface $X$ with automorphism group $\Delta / \Delta(\wp) \cong \mathrm{PSL}_{2} \mathbb{F}_{q}$, definable over the splitting subfield of $p$ in $k$, that is, over $\mathbb{Q}$ when $p \not \equiv \pm 1 \bmod 7$, and over $k$ when $p \equiv \pm 1 \bmod 7$. In the latter case, $q=p$, and the Galois conjugate curves $X^{\sigma}$ $($ for $\sigma \in \mathrm{Gal} k / \mathbb{Q})$ have the surface groups $\Delta(\sigma(\wp))$.

The former case applies in particular when $p=7$, the ramified prime over the ideal $\wp=(2-2 \cos 2 \pi / 7) \cdot \mathcal{O}$ of norm $q=7$, and gives the Klein quartic, of genus 3 . The prime 2 is inert in $k$, generating $\wp=2 \mathcal{O}$ of norm $q=8$, giving the Macbeath curve, of genus 7. The first nontrivial Galois actions occur for the three curves with $q=p=13$, in genus 14 .

\section{New tools}

Another instrument making visible the interplay between group theory and Riemann surfaces is the use of the canonical representation of the automorphism group $G=$ Aut $X$ of the quasiplatonic surface $X$ on its $\mathbb{C}$-vector space of holomorphic $k$-differentials. Denote the character (trace) of such a representation by $\chi$, and call two such characters $\chi_{1}$ and $\chi_{2}$ equivalent, denoted by $\chi_{1} \sim \chi_{2}$, if one results from the other by composition $\chi_{1}=\chi_{2} \circ \alpha$ with an automorphism $\alpha$ of $G$.

Then we have (as a counterpart of the Main Observation in [27]) the following:

Theorem 3. Let $X_{1}, \ldots, X_{n}$ be a Galois invariant family of quasiplatonic surfaces, admitting regular dessins $\mathcal{D}_{1}, \ldots, \mathcal{D}_{n}$ with the same signature and isomorphic automorphism groups, and with inequivalent characters $\chi_{1}, \ldots, \chi_{n}$ on their respective vector spaces of $k$-differentials for some $k \in \mathbb{N}$. Suppose there is a Galois extension $K / \mathbb{Q}$ such that the values of the $\chi_{j}$ generate $K$ and such that $\mathrm{Gal} K / \mathbb{Q}$ acts transitively on the equivalence classes of the characters via

$$
\sigma: \chi_{j} \mapsto \sigma \circ \chi_{j} \sim \chi_{i} \quad \text { for some } i
$$

Then we have $n=[K: \mathbb{Q}]$, and the surfaces $X_{j}$ all have $K$ as minimal field of definition and form a Galois orbit under $\mathrm{Gal} K / \mathbb{Q}$.

Proof. To prove this, by Proposition 1 we need only show that $K$ is the moduli field. We note first that any $\sigma \in \mathrm{Gal} \overline{\mathbb{Q}} / \mathbb{Q}$ not only acts on the family of surfaces $X_{j}$ and the associated Belyı functions, but also induces an action on the family of vector spaces of $k$-differentials, and maps the common automorphism group onto an isomorphic one acting on the image curve. Also clearly if $\sigma$ fixes $K$ elementwise, then all characters are mapped to equivalent ones, and so all $X_{j}$ are fixed (because the other characters are inequivalent), and therefore the moduli field has to be contained in $K$.

On the other hand, transitivity of the action of $\mathrm{Gal} K / \mathbb{Q}$ shows that the moduli field cannot be a proper subfield of $K$. 
Another variant of Proposition 4 turns out to be particularly useful for the small genus examples to be discussed shortly. It is known that every triangle group $\Delta$ of signature $\langle p, p, r\rangle$ (that is, with $p=q$ ) is embeddable as a subgroup of index 2 in an extension group $\bar{\Delta}$ of signature $\langle 2, p, 2 r\rangle$; see [25].

Now suppose that the curve $X$ has a surface group with normaliser $\Delta$. Then on $X$ we have two regular dessins corresponding to the Belyu functions $\beta$ and $1-\beta$, or in other words, with exchanged vertex colours. Since in this case $\beta$ is not uniquely determined by $X$ and its ramification orders, it can happen that $\beta$ (and hence also the dessin) is definable not over the minimal field of definition of $X$, but over some quadratic extension of it; as an example take the first pair of genus 18 curves of the table in the next section.

We call two dessins renormalisations of each other if they correspond under this interchange $\beta \leftrightarrow 1-\beta$ or another transformation permuting the critical values of $\beta$.

Theorem 4. Let $\left\{X_{j} \mid j \in(\mathbb{Z} / p \mathbb{Z})^{*}\right\}$ be a family of quasiplatonic surfaces each with maximal regular dessins $\mathcal{D}_{j}$ and $\mathcal{D}_{j}^{\prime}$ of signature $\langle p, p, r\rangle$. Suppose that $\mathcal{D}_{j}$ and $\mathcal{D}_{j}^{\prime}$ are renormalisations of each other, with Belyı functions $\beta_{j}$ and $1-\beta_{j}$ on $X_{j}$, and that the dessins $\mathcal{D}_{j}:=H_{j} \mathcal{D}_{1}$ and $\mathcal{D}_{j}^{\prime}:=H_{j} \mathcal{D}_{1}^{\prime}$ form two orbits under Wilson's map operations $H_{j}$. Also suppose that the combined family of all pairs $\left(X_{j}, \beta_{j}\right)$ and $\left(X_{j}, 1-\beta_{j}\right)\left(\right.$ for $\left.j \in(\mathbb{Z} / p \mathbb{Z})^{*}\right)$ is invariant under the action of the absolute Galois group $\mathrm{Gal} \overline{\mathbb{Q}} / \mathbb{Q}$. Then the curves $X_{j}$ can be defined over a subfield $K$ of the cyclotomic field $\mathbb{Q}\left(\zeta_{p}\right)$, where $\zeta_{p}=e^{2 \pi i / p}$, and the $X_{j}$ form a single orbit under the action of the absolute Galois group. Here the subfield $K$ is the fixed field of the subgroup

$$
H:=\left\{j \in(\mathbb{Z} / p \mathbb{Z})^{*} \mid H_{j} \mathcal{D}_{1} \cong \mathcal{D}_{1}\right\}
$$

when we identify $(\mathbb{Z} / p \mathbb{Z})^{*}$ with the Galois group $\operatorname{Gal} \mathbb{Q}\left(\zeta_{p}\right) / \mathbb{Q}$ as usual.

Proof. This can be proved using the same ideas as in the proofs of Theorem 2 of [20] and Theorem 2 of this paper. Every Galois conjugation fixing $\mathbb{Q}\left(\zeta_{p}\right)$ element-wise either preserves or interchanges $\mathcal{D}_{j}$ and $\mathcal{D}_{j}^{\prime}$, and so fixes $X_{j}$ up to isomorphism. It follows that the moduli field (and hence, by Proposition 1, also the field of definition) of $X_{j}$ is contained in $\mathbb{Q}\left(\zeta_{p}\right)$. Study of the local behaviour of the generators of the automorphism group on vertices of the dessin then shows that the Wilson operator $H_{j}$ has the same effect on the dessins $\mathcal{D}_{j}$ and $\mathcal{D}_{j}^{\prime}$ as the Galois conjugation taking $\zeta_{p} \mapsto \zeta_{p}^{k}$ for $k j \equiv 1 \bmod p$, up to renormalisation.

Another way to distinguish Galois orbits of quasiplatonic curves is via the study of their unramified function field extensions, which is equivalent to the study of the subgroup lattices of their surface groups $\Gamma$. We will in fact restrict to the consideration of the finite sublattice of all subgroups $N$ normal in both $\Gamma$ and $\Delta$ such that the quotient $\Gamma / N$ is an elementary abelian $m$-group for a small prime $m$. Let $\Gamma^{m}$ denote the subgroup of $\Gamma$ generated by the $m$ th powers of all elements of $\Gamma$; then we have: 
Theorem 5. Let $\left\{\mathcal{D}_{i} \mid 1 \leq i \leq n\right\}$ be a Galois invariant family of regular dessins of signature $\langle p, q, r\rangle$ on quasiplatonic surfaces $X_{i}=\Gamma_{i} \backslash \mathbb{H}$, such that $\mathcal{D}_{i}$ has automorphism group $\Delta / \Gamma_{i}$, where $\Delta$ is the $\langle p, q, r\rangle$ triangle group and $\Gamma_{i}$ is the surface group of $\mathcal{D}_{i}$. Let $m$ be a prime and for each $i$, let $\mathcal{L}_{i}$ be the lattice of normal subgroups $N$ of the triangle group $\Delta$ with the property that

$$
\left[\Gamma_{i}, \Gamma_{i}\right] \Gamma_{i}^{m} \leq N \leq \Gamma_{i}
$$

If these lattices $\mathcal{L}_{i}$ are pairwise non-isomorphic, then the dessins $\mathcal{D}_{i}$ can be defined over $\mathbb{Q}$.

In practice, often only the 'top' part of each lattice needs to be considered, in order to demonstrate non-isomorphism. Normal subgroups of small index in a finitely-presented group (such as the triangle group $\Delta$ ) can be found using the LowIndexNormalSubgroups procedure in the MAGMA system [3]. When $|\Delta / \Gamma|$ is small, that can sometimes be enough, but usually (and for larger cases) it is necessary to dig deeper, for example as follows.

For a surface $X=\Gamma \backslash \mathbb{H}$ of genus $g$, the group $\Gamma$ has a presentation in terms of $2 g$ generators and a single defining relator (which can be written as a product of $g$ commutators), so its abelianisation $\Gamma /[\Gamma, \Gamma]$ has rank $2 g$. Hence, in particular, the largest abelian quotient of $\Gamma$ of exponent $m$ is $\Gamma /[\Gamma, \Gamma] \Gamma^{m}$, which is isomorphic to $\left(C_{m}\right)^{2 g}$. In between $\Gamma$ and $[\Gamma, \Gamma] \Gamma^{m}$ there may be other normal subgroups of $\Delta$. Again, for small values of $g$ and $|\Delta / \Gamma|$ (and $m$ ), these can sometimes be found using a combination of the Reidemeister-Schreier process and the LowIndexNormalSubgroups procedure in MAGMA [3]; in other cases, they may often be found by investigating the submodule structure of the quotient $\Gamma /[\Gamma, \Gamma] \Gamma^{m}$ as a $\Delta / \Gamma$-module.

If the numbers of such 'intermediate' normal subgroups are different for different choices of $\Gamma$, then the corresponding lattices are non-isomorphic, in which case Theorem 5 may be applied.

Another interpretation of the subgroups used in Theorem 5 will play a major role in Section 5. Recall that $\Gamma$ is isomorphic to $\pi_{1}(X)$ and $\Gamma /[\Gamma, \Gamma] \Gamma^{m}$ is isomorphic to the $\bmod m$ homology group $H_{1}(X, \mathbb{Z} / m \mathbb{Z})$, so the normal subgroups $N$ in question correspond to the (Aut $X$ )-invariant submodules of this homology group.

\section{The classification}

A complete classification of all regular maps and hypermaps of small genera is described in [6], with the maps and hypermaps themselves available on the first author's website. In the following table, we restrict our consideration to maximal regular dessins on quasiplatonic curves of genera from 2 to 18, and omit all cases for which Theorem 1 or Proposition 3 applies. Thus our table begins with genus $g=7$. 
In the 3rd to 6th columns of this table, 'Order' means the order of the automorphism group of the dessin (and by maximality, also of the curve), 'Number' denotes the number of non-isomorphic curves of the kind described by the current row, and 'Field $X$ ' means the minimal field of definition for the curve, while 'Field $\mathcal{D}$ ' means the minimal field of definition of the dessins. The final column gives a number for reference to the relevant comment in the explanations that follow the table.

For all curves in question, the table contains the maximal dessin only, corresponding to the (unique) triangle group $\Delta$ that normalises the surface group $\Gamma$. In most cases, the entries in the signature $\langle p, q, r\rangle$ are pairwise distinct, in which case the moduli field (and hence also the field of definition) for the dessin is the same as that for the curve; see Lemma 5 of [35]. In all other cases, two of the entries coincide, and then the triangle group of signature $\langle p, p, r\rangle$ is a subgroup of index 2 in another one of signature $\langle 2, p, 2 r\rangle$ (see [25], [29]), and the underlying surfaces are pairwise isomorphic by conjugation of their surface groups in this extension. Geometrically, the respective dessins are related to each other by renormalisation, that is duality or transposition of vertex colours. In that case it may happen that the Belyı functions can be defined only over a quadratic extension $k(\sqrt{\alpha})$ of the minimal field of definition $k$ of $X$; see the proof of Theorem 4 above. For example, if both dessins result from each other by an transposition of vertex colours, $\beta$ is defined over a quadratic extension $k(\sqrt{\alpha})$ if and only if $1-\beta=\beta^{\sigma}$ for the nontrivial Galois conjugation $\sigma$ of $k(\sqrt{\alpha}) / k$. For several curves we were unable to decide whether we are in this situation; in these cases we put a question mark in the column "Field $\mathcal{D}$ ".

In the table we always display the signature triples so that $p \leq q \leq r$, since the order of the entries is irrelevant for the triangle group and the curve. For the Belyu function and the dessin, reordering means renormalisation and duality or triality. However, one should note that for the application of Wilson operators, the ordering of the entries $p, q$ and $r$ in the signature is essential. If it happens that for a given triple consisting of the genus, signature (for a maximal dessin), and automorphism group, there is a unique pair of dessins, both chiral, then the two dessins must be defined over an imaginary quadratic number field, and these two dessins are transposed by the Wilson operator $H_{-1}$. In that case such a Wilson operator can be found for any ordering of $p, q, r$, but this does not always correspond to a Galois conjugation of the dessin, so Proposition 4 or Theorem 2 cannot be applied immediately. For example, in the case of comment 5 below (for genus 10 and order 432), the application of $H_{-1}$ to the signature $\langle 2,3,8\rangle$ gives a Galois conjugation in $\mathbb{Q}(\sqrt{-3})$, while application to the signature $\langle 2,8,3\rangle$ would give a Galois conjugation in $\mathbb{Q}(\sqrt{-1})$ or $\mathbb{Q}(\sqrt{-2})$. Accordingly, a direct check is necessary to choose the correct ordering.

Unless otherwise stated, all group theoretic calculations were made with the help of the MAGMA system [3]. 


\begin{tabular}{|c|c|c|c|c|c|c|}
\hline$g$ & Signature & Order & Number & Field $X$ & Field $\mathcal{D}$ & Comment \\
\hline \hline 7 & 269 & 54 & 2 & $\mathbb{Q}(\sqrt{-3})$ & $\mathbb{Q}(\sqrt{-3})$ & 1 \\
& 237 & 504 & 1 & $\mathbb{Q}$ & $\mathbb{Q}$ & 2 \\
\hline 8 & 238 & 336 & 2 & $\mathbb{Q}(\sqrt{2})$ & $\mathbb{Q}(\sqrt{2})$ & 3 \\
& 266 & 84 & 2 & $\mathbb{Q}(\sqrt{-3})$ & $?$ & 4 \\
\hline 10 & 238 & 432 & 2 & $\mathbb{Q}(\sqrt{-3})$ & $\mathbb{Q}(\sqrt{-3})$ & 5 \\
& 3614 & 42 & 2 & $\mathbb{Q}(\sqrt{-3})$ & $\mathbb{Q}(\sqrt{-3})$ & 6 \\
\hline 11 & 248 & 160 & 2 & $\mathbb{Q}(\sqrt{-1})$ & $\mathbb{Q}(\sqrt{-1})$ & 7 \\
& 248 & 160 & 2 & $\mathbb{Q}(\sqrt{-1})$ & $\mathbb{Q}(\sqrt{-1})$ & 7 \\
& 2412 & 120 & 2 & $\mathbb{Q}(\sqrt{-1})$ & $\mathbb{Q}(\sqrt{-1})$ & 6 \\
\hline 12 & 2510 & 110 & 4 & $\mathbb{Q}\left(\zeta_{5}\right)$ & $\mathbb{Q}\left(\zeta_{5}\right)$ & 5 \\
\hline 13 & 346 & 96 & 2 & $\mathbb{Q}(\sqrt{-3})$ & $\mathbb{Q}(\sqrt{-3})$ & 6 \\
& 448 & 64 & 2 & $\mathbb{Q}$ & $?$ & 8 \\
& 448 & 64 & 2 & $\mathbb{Q}$ & $?$ & 8 \\
\hline 14 & 237 & 1092 & 3 & $\mathbb{Q}\left(2 \cos \frac{2 \pi}{7}\right)$ & $\mathbb{Q}\left(2 \cos \frac{2 \pi}{7}\right)$ & 9 \\
& 266 & 156 & 2 & $\mathbb{Q}(\sqrt{-3})$ & $?$ & 4 \\
\hline 15 & 2312 & 336 & 2 & $\mathbb{Q}(\sqrt{-3})$ & $\mathbb{Q}(\sqrt{-3})$ & 6 \\
& 336 & 168 & 2 & $\mathbb{Q}(\sqrt{-3})$ & $?$ & 4 \\
& 5511 & 55 & 2 & $\mathbb{Q}(\sqrt{5})$ & $\mathbb{Q}\left(\zeta_{5}\right)$ & 6 \\
\hline 16 & 2618 & 108 & 2 & $\mathbb{Q}(\sqrt{-3})$ & $\mathbb{Q}(\sqrt{-3})$ & 6 \\
& 346 & 120 & 2 & $\mathbb{Q}(\sqrt{6})$ & $\mathbb{Q}(\sqrt{6})$ & 10 \\
& 3612 & 72 & 2 & $\mathbb{Q}$ & $\mathbb{Q}$ & 11 \\
& 4612 & 60 & 2 & $\mathbb{Q}(\sqrt{-1})$ & $\mathbb{Q}(\sqrt{-1})$ & 5 \\
\hline 17 & 237 & 1344 & 2 & $\mathbb{Q}(\sqrt{-3})$ & $\mathbb{Q}(\sqrt{-3})$ & 5 \\
& 246 & 384 & 2 & $\mathbb{Q}(\sqrt{-1})$ & $\mathbb{Q}(\sqrt{-1})$ & 5 \\
& 2714 & 112 & 2 & $\mathbb{Q}(\sqrt{-7})$ & $\mathbb{Q}(\sqrt{-7})$ & 5 \\
& 344 & 192 & 2 & $\mathbb{Q}(\sqrt{-1})$ & $?$ & 12 \\
& 446 & 96 & 2 & $\mathbb{Q}$ & $?$ & 13 \\
\hline
\end{tabular}

\section{Comments on the table:}

1) Application of Proposition 4 to the signature $\langle 2,9,6\rangle$, with the subgroup $H:=\{1,13,7\}$ of $(\mathbb{Z} / 18 \mathbb{Z})^{*}$ fixing the proper subfield $\mathbb{Q}(\sqrt{-3})$ of $\mathbb{Q}\left(\zeta_{18}\right)$.

2) The Macbeath curve of genus 7 of signature $\langle 2,3,7\rangle$ with automorphism group $\mathrm{PSL}_{2} \mathbb{F}_{8}$. This is the Hurwitz curve of second smallest genus. It should not really be contained in this table (since by Proposition 6 it is uniquely determined, with a dessin defined over $\mathbb{Q}$ ), but we mention it here for two 
good reasons: firstly, there is still no model known for it that is given by equations with rational coefficients, and secondly, Macbeath's curve has two non-maximal chiral dessins of type $\langle 7,2,7\rangle$, called the Edmond maps, coming from the two non-isomorphic regular embeddings of the complete graph $K_{8}(\text { see }[16])^{1}$. Again Proposition 4 applies here, with the subgroup $H:=\{1,9,11\}$ of $(\mathbb{Z} / 14 \mathbb{Z})^{*}$ giving the field of definition $\mathbb{Q}(\sqrt{-7})$. As maps, these two dessins are dual to each other. However, this is not the lowest genus for which two regular non-isomorphic Galois conjugate dessins exist on the same quasiplatonic curve: on Klein's quartic in genus 3 there are two complex conjugate dessins of signature $\langle 3,3,7\rangle$; see Theorem 1 of [28]. By Proposition 4 , they are defined over $\mathbb{Q}(\sqrt{-3})$.

3) In this case, both surface groups are principal congruence subgroups of a norm 1 group in a quaternion algebra defined over the centre field $\mathbb{Q}(\sqrt{2})$, and the levels are the two Galois conjugate ideals lying over 7 , both with residue class field $\mathbb{F}_{7}$. Since the triangle group of signature $\langle 2,3,8\rangle$ is an index 2 extension of the norm 1 group [29], the quotient by the surface group is $\mathrm{PGL}_{2} \mathbb{F}_{7}$. In an analogous way to Proposition 6 , these two curves should therefore be defined over $\mathbb{Q}(\sqrt{2})$ and be Galois conjugate, but it is difficult to translate the proof of Proposition 6 to this case. Theorem 3, however, gives an easier way. Via a calculation in GAP [13] using Eichler's trace formula, one may see that the characters on the spaces of holomorphic 2-differentials decompose into irreducible constituents as $\chi_{4}^{i}+\chi_{5}^{0}+\chi_{6}^{0}$ for $i=0,1$ : following ATLAS notation [7], $\chi_{4}^{0}$ and $\chi_{4}^{1}$ are the algebraically conjugate extensions to $\mathrm{PGL}_{2} \mathbb{F}_{7}$ of the irreducible character $\chi_{4}$ of degree 6 of $\mathrm{PSL}_{2} \mathbb{F}_{7}$, while $\chi_{5}^{0}$ and $\chi_{6}^{0}$ are the extensions of the irreducible characters $\chi_{5}$ and $\chi_{6}$ of degrees 7 and 8 of $\mathrm{PSL}_{2} \mathbb{F}_{7}$, defined by taking values 1 and -1 (rather than -1 and 1 ) on the class $6 \mathrm{~A}$ of elements of order 6 . The values of the characters $\chi_{5}^{0}$ and $\chi_{6}^{0}$ are all rational, whereas those of each $\chi_{4}^{i}$ generate $\mathbb{Q}(\sqrt{2})$, so the two curves are defined over this field and are conjugate under its Galois group.

4) Direct application(s) of Theorem 4 . Note that the genus 8 curves with signature $\langle 2,6,6\rangle$ are unramified degree 7 cyclic covers of the genus 2 curve $y^{2}=x^{6}-1$. For this and for the next comment, see also Section 5 .

5) Direct applications of Proposition 4. Note that the genus 12 dessins of type $\langle 2,5,10\rangle$ are regular embeddings of the complete graph $K_{11}$ (see [20]), and form two chiral pairs. The genus 17 curves of signature $\langle 2,3,7\rangle$ are degree 8 coverings of Klein's quartic, with covering group $C_{2}^{3}$ (see [23]).

6) Applications of Proposition 4 to reorderings of the respective signatures. In genus 10 take $\langle 3,14,6\rangle$; the dessins are invariant under $H_{j}$ for $j \equiv 1 \bmod 3$, and are interchanged by $H_{-1}$. Similarly, consider the signatures $\langle 2,12,4\rangle$ in genus $11,\langle 3,6,4\rangle$ in genus 13 , and $\langle 2,12,3\rangle$ and $\langle 5,11,5\rangle$ in genus 15 . In this last case, the dessins are permuted by the Wilson operators $H_{j}$ for

\footnotetext{
${ }^{1}$ Added in proof (September 2012): Rubén Hidalgo kindly informed us that there is now a relatively simple equation over $\mathbb{Q}$ available, found by Bradley Brock, and Hidalgo worked out explicit versions of the Bely̆ functions for the Edmond maps.
} 
units $j \bmod 5$, and the curves are invariant under $H_{j}$ for $j \equiv \pm 1 \bmod 5$ and interchanged by $H_{j}$ for $j \equiv \pm 2 \bmod 5$ - see also Theorem 1 of [27]. This is the only case in the table where (on both curves) one has two chiral dessins living on the same curve. Finally, for the first line in genus 16 take $\langle 2,18,6\rangle$.

7) These two cases in genus 11 have different automorphism groups of the same order. In both cases, Proposition 4 can be applied to the signature $\langle 2,8,4\rangle$, with all four dessins invariant under $H_{j}$ for $j \equiv 1,5 \bmod 8$.

8) These are two pairs of regular dessins having the same signature but nonisomorphic automorphism groups. Moreover, the dessins with the same automorphism group can be distinguished by the number of normal subgroups $N$ of $\Delta$ contained in the surface group $\Gamma$ such that $\Gamma / N$ is an elementary abelian 3-group. For one pair, a MAGMA-assisted calculation shows there are such normal subgroups of index $3^{2 i}$ in $\Gamma$ for all $i \in\{0,1,2, \ldots, 13\}$ in one case, while in the other case, the only such $N$ have index $3^{2 i}$ for $i \in\{0,1,4,5,8,9,12,13\}$. For the second pair, in both cases there are such normal subgroups of index $3^{2 i}$ in $\Gamma$ for all $i \in\{0,1,2, \ldots, 13\}$, but one has exactly 2480 possibilities for $N$, while the other has only 1294 . Hence by Theorem 5, the dessins are not Galois conjugate.

9) Application of Proposition 6.

10) In this case the two surface groups are congruence subgroups of a norm 1 group in a quaternion algebra with centre field $\mathbb{Q}(\sqrt{6})$, generated by the triangle group; the norm 1 group is a quadrangle group of index 2 (see [29]), and the levels are the two prime ideals above 5, and are Galois conjugate under $\sqrt{6} \mapsto-\sqrt{6}$. Unfortunately, methods like those used for the proof of Proposition 6 do not seem to be sufficient for a proof that both curves and dessins are defined over $\mathbb{Q}(\sqrt{6})$. (Work in progress by Pete Clark and John Voight about congruence subgroups of triangle groups seems to be more successful for this aim.) We can prove it, however, by the following argument. The surface groups arise as kernels of two homomorphisms $h_{1}, h_{2}$ onto the symmetric group $S_{5}$. Since $S_{5}$ contains five conjugate subgroups of index 5 isomorphic to $S_{4}$, one may consider the preimages $P_{1}, P_{2}$ under $h_{1}, h_{2}$ of $S_{4}$, which are uniquely determined up to conjugation. These subgroups $P_{j}$ are of genus 0 , and the corresponding dessins $\mathcal{N}_{j}$ are given in Figure 2.
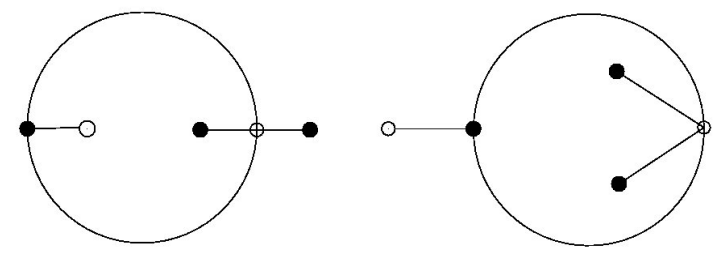

Figure 2. Two genus 0 dessins of type $\langle 4,3,6\rangle$, up to homeomorphism. 
One may observe that up to isomorphism these are the only genus 0 dessins with five edges on which two permutations of order 3 and 4 act such that their product is a permutation of order 6 . Applying a Möbius transformation, we may assume that in both cases the face centres are 0 and $\infty$, and that the right white vertex, which is an order 4 zero of the Belyu function, is situated at $z=1$. If $a$ is the left white vertex (an order 1 zero), then the Belyu function must have the form

$$
\beta(z)=\frac{k(z-1)^{4}(z-a)}{z^{3}} .
$$

Since $\beta-1$ has a triple zero at the black vertex $b$ on the left hand side of the dessin, one expects a double zero of $\beta^{\prime}$ outside of $z=0$ or 1 , giving a condition on $a$ and hence also the value of $b$ :

$$
a=-10 \pm 4 \sqrt{6} \text { and } b=-3 \pm \sqrt{6}
$$

Moreover, the condition $\beta(b)=1$ gives an obvious value of $k \in \mathbb{Q}(\sqrt{6})$. Finally, the two regular dessins of genus 16 can be considered as the unique regular coverings of the $\mathcal{N}_{j}$ of smallest degree, and then since the Galois conjugation $\sqrt{6} \mapsto-\sqrt{6}$ extends from $\mathcal{N}_{1} \mapsto \mathcal{N}_{2}$ to these regular covers, the result follows.

11) This case is similar to that in Comment 8). A MAGMA-assisted calculation shows that there are different numbers of normal subgroups $N$ of $\Delta$ contained in the surface group $\Gamma$ such that $\Gamma / N$ is an elementary abelian 2-group. Hence, by Theorem 5, the dessins are not Galois conjugate.

12) In this case there are four dessins living on two non-isomorphic curves. The pairs of dessins living on the same curves can be transformed into each other by passing to a dual dessin, or by conjugation in the triangle group $\langle 2,4,6\rangle$, or by applying the Petrie duality operator $P=H_{1,-1}$ : the Petrie paths of one dessin are the face boundaries of the other. On the other hand, the dessins on non-isomorphic curves are interchanged by the generalised Wilson operator $H_{-1}=H_{-1,-1}$, applied to the signature $\langle 4,4,3\rangle$, and then Theorem 4 applies.

13) These two cases are similar to the case in Comment 8) above; both can be handled by considering normal subgroups $N$ of $\Delta$ contained in the surface group $\Gamma$ such that $\Gamma / N$ is an elementary abelian 3-group.

14) An application of Proposition 4 again. Here we have four maximal dessins $\mathcal{D}$ of type $\langle 2,8,8\rangle$, on two non-isomorphic curves, permuted transitively by the Wilson operators $H_{j}$ for $j \in(\mathbb{Z} / p \mathbb{Z})^{*}$. These curves $X$ are the first of an infinite series of chiral pairs of quasiplatonic surfaces constructed in $\S 3$, Example (iii) of [1]; see Theorem 3.1 in [5] for the associated maps. The dessins $\mathcal{D}$ are all 17-sheeted regular unbranched coverings of a dessin $\overline{\mathcal{D}}$ of type $\langle 2,8,8\rangle$ on the Riemann surface $\bar{X}$ of genus 2 given as an affine curve by $y^{2}=x^{5}-x$, a special case of a general construction treated in some more detail in the final section. 
15) As in Comment 12), there are four dessins on two non-isomorphic curves. We can apply the generalized Wilson operators $H_{i, j}$ to the four dessins living on two non-isomorphic curves. The dessins are pairwise equivalent under exchange of the vertex colours (or conjugation in the triangle group $\langle 2,14,42\rangle$, or under a generalised Wilson operator, say $\left.H_{1,9}\right)$. All dessins are invariant up to isomorphism under the action of the Wilson operators $H_{i}=H_{i, i}$ for $i$ coprime to 14. By Proposition 5, the fixed fields in question are all equal to $\mathbb{Q}$.

\section{Problems, conjectures, and infinite families}

One may of course continue this kind of calculation, and in certain genera this can be easy: in genus $g=20$ we have - besides those cases treated by means of Theorem 1 or Proposition 3 - to consider just one such pair of maximal regular dessins: they have signature $\langle 2,6,6\rangle$ with automorphism groups of order 228 on curves defined over $\mathbb{Q}(\sqrt{-3})$. If on the other hand $g-1$ has a complicated prime decomposition, we can expect more and more complicated situations for which involved calculations are needed, like those in Comment 8) or 10).

For the moment, it seems not to be worthwile to continue this way. In these "small" genera, the degree of the fields of definition seems to grow so slowly that very large genera might be necessary to find examples for which, say, non-abelian number fields are needed for the definition of the curves. Several results from the last ten years, such as those in [27] or [20], show that the degrees of these minimal fields of definition for quasiplatonic curves are in fact unbounded; on the other hand it is unknown whether the absolute Galois group acts faithfully on the set of all regular dessins (a question raised by Gabino González-Díez)2 . And up to now we do not have a single explicit example of a quasiplatonic curve with a non-abelian field of moduli.

However, some of the regular dessins of small genera give an interesting insight into those of higher genera: they represent the first examples of infinite families showing certain similarities under the action of the absolute Galois group.

For each of the types $\langle 2, q, r\rangle=\langle 2,5,10\rangle,\langle 2,6,6\rangle$ and $\langle 2,8,8\rangle$, the dessins $\mathcal{D}$ of that type in the table, together with the pair of type $\langle 2,6,6\rangle$ and genus 20 mentioned above, are members of an infinite family of $p$-sheeted unbranched coverings of a regular genus 2 dessin $\overline{\mathcal{D}}$ of that type, for all primes $p$ in a certain arithmetic progression. The corresponding quasiplatonic surfaces $X$, of genus $p+1$, are constructed in $\S 3$, Examples (i)-(iii) [1], and the associated maps, regarded by duality as dessins of type $\langle q, 2, r\rangle$, are studied in Theorem 3.1 of [5].

For each of these three types, the dessin $\overline{\mathcal{D}}$ corresponds to a torsion-free normal subgroup $\bar{\Gamma}$ of finite index in the triangle group $\Delta$ of that type, with $\Delta / \bar{\Gamma} \cong A:=$ Aut $\overline{\mathcal{D}}$. Since the surface $\bar{X}$ underlying $\overline{\mathcal{D}}$ has fundamental group $\pi_{1}(\bar{X}) \cong \bar{\Gamma}$, the

\footnotetext{
${ }^{2}$ Added in proof (September 2012): a positive answer to this question has been announced by Gabino González-Díez and Andrei Jaikin-Zapirain.
} 
$\bmod p$ homology group $H_{1}(\bar{X} ; \mathbb{Z} / p \mathbb{Z})$ is isomorphic to $\bar{\Gamma} /[\bar{\Gamma}, \bar{\Gamma}] \bar{\Gamma}^{p}$, as already mentioned at the end of Section 3. This isomorphism is an isomorphism of $A$-modules, where the action of $A$ on $H_{1}(\bar{X} ; \mathbb{Z} / p \mathbb{Z})$ is induced by its natural action on $\bar{X}$, while its action on $\bar{\Gamma} /[\bar{\Gamma}, \bar{\Gamma}] \bar{\Gamma}^{p}$ is induced by the action of $\Delta$ by conjugation on $\bar{\Gamma}$.

The action of $A$ on $\overline{\mathcal{D}}$ allows one to deduce its action on $H_{1}(\bar{X} ; \mathbb{Z} / p \mathbb{Z}$ ) (see Mustafa Kazaz's PhD thesis [21] for full details). In particular, if $e$ is the exponent of $A$ then, for each prime $p \equiv 1 \bmod e$, this module is a direct sum of four 1-dimensional $A$-submodules. It follows that $\bar{\Gamma}$ has four subgroups $\Gamma$ of index $p$ which are normal in $\Delta$, with the $A$-modules $\bar{\Gamma} / \Gamma$ isomorphic to these summands. These subgroups $\Gamma$ correspond to four nonisomorphic regular dessins $\mathcal{D}$, all of type $\langle 2, q, r\rangle$ and genus $p+1$, for which Aut $\mathcal{D}$ is a split extension of a normal subgroup $P \cong C_{p}$ by $A$, with $\mathcal{D} / P \cong \overline{\mathcal{D}}$. (Primes $p \not \equiv 1 \bmod e$ yield dessins of genus $p^{d}+1$ as $p^{d}$-sheeted coverings of $\bar{D}$, for various $d \leq 4$.)

The simplest case concerns the dessins of type $\langle 2,5,10\rangle$. Here the quotient dessin $\overline{\mathcal{D}}$, corresponding to the commutator subgroup $\bar{\Gamma}=[\Delta, \Delta]$ of $\Delta$, is formed by identifying opposite sides of a decagon, colouring the resulting two 5 -valent vertices black, and regarding the midpoints of the five edges as white vertices of valency 2 . The underlying surface $\bar{X}$ is given as an affine curve by $x^{5}+y^{2}=1$, and the Bely function is $(x, y) \mapsto y^{2}$, so that the white vertices are at the points $(x, 0)$ with $x^{5}=1$, and the black vertices are at $(0, \pm 1)$. We have $A=\operatorname{Aut} \overline{\mathcal{D}} \cong \Delta / \bar{\Gamma} \cong C_{10}$, generated by a rotation $\alpha$ of the single face through $\pi / 5$, or equivalently by $(x, y) \mapsto\left(\zeta_{5} x,-y\right)$. For each prime $p \equiv 1 \bmod 10$, the module $H_{1}(\bar{X} ; \mathbb{Z} / p \mathbb{Z})$ is a direct sum of four 1-dimensional $A$-submodules on which the eigenvalues $\lambda$ of $\alpha$ are the four primitive 10 th roots of 1 in $(\mathbb{Z} / p \mathbb{Z})^{*}$. It follows that there are four subgroups $\Gamma=\Gamma_{\lambda}$ of index $p$ in $\bar{\Gamma}$, each normal in $\Delta$, with the generator of $\Delta / \bar{\Gamma}$ corresponding to $\alpha$ having these eigenvalues $\lambda$ on the quotients $\bar{\Gamma} / \Gamma_{\lambda}$. The corresponding dessins $\mathcal{D}_{\lambda}$ have type $\langle 2,5,10\rangle$ and genus $p+1$. These roots $\lambda$ are permuted transitively by the operations $\lambda \mapsto \lambda^{j}$ for $j \in(\mathbb{Z} / 10 \mathbb{Z})^{*}$, so the dessins $\mathcal{D}_{\lambda}$ form a single orbit under the corresponding Wilson operators $H_{j}$, consisting of two chiral pairs $\mathcal{D}_{\lambda^{ \pm 1}}$. As the only regular dessins with their type and automorphism group, the four dessins $\mathcal{D}_{\lambda}$ form a Gal $\overline{\mathbb{Q}} / \mathbb{Q}$-invariant family, so Proposition 4 shows that they and their underlying curves $X_{\lambda}$ each form a single orbit of Gal $\overline{\mathbb{Q}} / \mathbb{Q}$, and are defined over $\mathbb{Q}\left(\zeta_{10}\right)=\mathbb{Q}\left(\zeta_{5}\right)$. The triangle group $\Delta$ of type $\langle 2,5,10\rangle$, as a maximal Fuchsian group, is the normaliser of each $\Gamma_{\lambda}$ in $\mathrm{PSL}_{2} \mathbb{R}$, so the four curves $X_{\lambda}$ are mutually nonisomorphic. Since $\operatorname{Gal} \mathbb{Q}\left(\zeta_{5}\right) / \mathbb{Q}$ has order 4 , it follows that $\mathbb{Q}\left(\zeta_{5}\right)$ is the minimal field of definition of both the curves and the dessins. The first example of this series occurs when $p=11$, giving the genus 12 entry in the table; if we use a renormalisation to regard these dessins as having type $\langle 10,2,5\rangle$, that is, as 10-valent pentagonal maps, we obtain the four regular embeddings of the complete graph $K_{11}$ described as maps in [16] and as a Galois orbit in [20].

When the type is $\langle 2,8,8\rangle$, the dessin $\overline{\mathcal{D}}$ lies on the curve $\bar{X}$ of genus 2 given by $y^{2}=x^{5}-x$, with Belyu function $(x, y) \mapsto 1-x^{4}$. One can form $\overline{\mathcal{D}}$ by identifying opposite sides of an octahedron to give a map with one vertex, one face and four edges; this single vertex, corresponding to the point $(0,0) \in \bar{X}$, is coloured black, and the midpoints of the four edges are regarded as white vertices of valency 2 , 
where $x^{4}=1$ and $y=0$. A rotation $\alpha:(x, y) \mapsto\left(i x, \zeta_{8} y\right)$ of the octagon through $\pi / 4$ generates $A=$ Aut $\overline{\mathcal{D}} \cong \Delta / \bar{\Gamma} \cong C_{8}$, where $\bar{\Gamma}$ is the unique torsion-free normal subgroup of $\Delta$ with this quotient. If $p \equiv 1 \bmod 8$ then $H_{1}(\bar{X} ; \mathbb{Z} / p \mathbb{Z})$ is a direct sum of four 1-dimensional $A$-submodules, on which the eigenvalues $\lambda$ of $\alpha$ are the four primitive 8 th roots of 1 in $(\mathbb{Z} / p \mathbb{Z})^{*}$. We thus obtain four subgroups $\Gamma_{\lambda}$ of index $p$ in $\bar{\Gamma}$, each normal in $\Delta$, such that $\alpha$ has eigenvalue $\lambda$ on $\bar{\Gamma} / \Gamma_{\lambda}$. The regular dessin $\mathcal{D}_{\lambda}$ corresponding to $\Gamma_{\lambda}$ has type $\langle 2,8,8\rangle$ and genus $p+1$, with Aut $\mathcal{D}_{\lambda}$ a split extension of $C_{p}$ by $C_{8}$. As in the preceding case, these four dessins form a single orbit under the Wilson operators $H_{j}$ for $j \in(\mathbb{Z} / 8 \mathbb{Z})^{*}$, consisting of two chiral pairs $\mathcal{D}_{\lambda^{ \pm 1}}$. The maximal triangle group of type $\langle 2,4,8\rangle$ contains $\Delta$ with index 2 , transposing its generators $\gamma_{1}$ and $\gamma_{\infty}=\left(\gamma_{0} \gamma_{1}\right)^{-1}$ of order 8 by conjugation; since each of these acts by conjugation on each $\bar{\Gamma} / \Gamma_{\lambda}$ as the cube of the other, $H_{3}$ transposes each $\mathcal{D}_{\lambda}$ with a dual dessin $\mathcal{D}_{\lambda^{3}}$ on an isomorphic curve. It follows from Proposition 4 that the four dessins and their two underlying curves each form a single Galois orbit, the minimal field of definition of the dessins is $\mathbb{Q}\left(\zeta_{8}\right)$, and for the curves it is the subfield $\mathbb{Q}(\sqrt{-2})$ fixed by the automorphism $\zeta_{8} \mapsto \zeta_{8}^{3}$.

If the type is $\langle 2,6,6\rangle$ then $\bar{X}$ is given by $x^{6}+y^{2}=1$, with Belyu function $(x, y) \mapsto y^{2}$. The dessin $\overline{\mathcal{D}}$ is a double covering of a dessin $\tilde{\mathcal{D}}$ on the Riemann sphere consisting of a black vertex at 0 joined by straight line segments to white vertices at the sixth roots of 1 , where the covering $\overline{\mathcal{D}} \rightarrow \tilde{\mathcal{D}}$ is branched. In this case $A \cong C_{2} \times C_{6}$, the two direct factors being generated by automorphisms $\alpha:(x, y) \mapsto(x,-y)$ and $\beta:(x, y) \mapsto\left(\zeta_{6} x, y\right)$, respectively transposing the two sheets and rotating them through $\pi / 3$. For each prime $p \equiv 1 \bmod 6$ we obtain four normal subgroups $\Gamma_{\lambda}$ of $\Delta$, of index $p$ in $\bar{\Gamma}$, such that $\alpha$ has eigenvalue -1 on each $\bar{\Gamma} / \Gamma_{\lambda}$, while the eigenvalues $\lambda$ of $\beta$ are the four elements of order 3 or 6 in $(\mathbb{Z} / p \mathbb{Z})^{*}$. The corresponding dessins $\mathcal{D}_{\lambda}$ form two chiral pairs $\mathcal{D}_{\lambda^{ \pm 1}}$; however, unlike the two preceding cases, here they do not form a single orbit under the Wilson operations $H_{j}$. The maximal triangle group of type $\langle 2,4,6\rangle$ contains $\Delta$ with index 2 , transposing its generators $\gamma_{1}$ and $\gamma_{\infty}$ by conjugation. These induce the automorphisms $\beta$ and $(\alpha \beta)^{-1}$, which have eigenvalues $\lambda$ and $-\lambda^{-1}$, so there are two dual pairs $\mathcal{D}_{\lambda}, \mathcal{D}_{-\lambda^{-1}}$, each pair lying on one of two nonisomorphic curves. By applying a renormalisation $\beta \mapsto 1 / \beta$ we may regard these dessins as having type $\langle 6,6,2\rangle$, in which case Theorem 4 shows that the curves are Galois conjugate, with $\mathbb{Q}\left(\zeta_{6}\right)=\mathbb{Q}(\sqrt{-3})$ as their minimal field of definition. Unfortunately our methods do not provide corresponding results for the dessins.

\section{References}

[1] Belolipetsky, M. And Jones, G. A.: Automorphism groups of Riemann surfaces of genus $p+1$, where $p$ is prime. Glasg. Math. J. 47 (2005), 379-393.

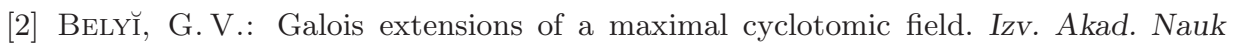
SSSR Ser. Mat. 43 (1979), 267-276, 479.

[3] Bosma, W., Cannon, J. and Playoust, C.: The magma algebra system. I. The user language. J. Symbolic Comput. 24 (1997), 235-265. 
[4] Cohen, P. B., Itzykson, C. And Wolfart, J.: Fuchsian triangle groups and Grothendieck dessins. Variations on a theme of Belyı. Comm. Math. Phys. 163 (1994), 605-627.

[5] Conder, M. D. E., Širáň, J. And Tucker, T. W.:, The genera, reflexibility and simplicity of regular maps. J. Eur. Math. Soc. 12 (2010) 343-364.

[6] Conder, M.D.E.: Regular maps and hypermaps of Euler characteristic -1 to -200. J. Combin. Theory Ser. B 99 (2009), 455-459. Associated lists of computational data available at www.math.auckland.ac.nz/ conder/hypermaps.html.

[7] Conway, J. H., Curtis, R. T., Norton, S. P., Parker, R. A. and Wilson, R. A.: Atlas of finite groups. Clarendon Press, Oxford, 1985.

[8] Coombes, K. and Harbater, D.: Hurwitz families and arithmetic Galois groups. Duke Math. J. 52 (1985), 821-839.

[9] CoRI, R.: Un code pour les graphes planaires et ses applications. Astérisque 27, Soc. Math. de France, Paris, 1975.

[10] Dèbes, P. And Emsalem, M.: On fields of moduli of curves. J. Algebra 211 (1999), no. $1,42-56$.

[11] DžAmbić, A.: Macbeath's infinite series of Hurwitz groups. In Arithmetic and geometry around hypergeometric functions, 101-108. Progr. Math. 260, Birkhäuser, Basel, 2007.

[12] Feierabend, F.: Galois-Operationen auf verallgemeinerten Macbeath-Hurwitz Kurven. PhD thesis, Frankfurt, 2008.

[13] The Gap Group: GaP-groups, algorithms, and programming. Version 4.4.12, 2008. www . gap-system.org.

[14] Grothendieck, A.: Esquisse d'un programme. In Geometric Galois actions 1, 5-48. London Math. Soc. Lecture Note Ser. 242, Cambridge Univ. Press, Cambridge, 1997.

[15] Hidalgo, R.: Homology closed Riemann surfaces. Quarterly J. Math. 63 (2012), 931-952.

[16] James, L. D. And Jones, G. A.: Regular orientable imbeddings of complete graphs. J. Combin. Theory Ser. B 39 (1985), 353-367.

[17] Jones, G. A. And Singerman, D.: Theory of maps on orientable surfaces. Proc. London Math. Soc. (3) 37 (1978), 273-307.

[18] Jones, G. A. And Singerman, D.: Belyı̆ functions, hypermaps and Galois groups. Bull. London Math. Soc. 28 (1996), 561-590.

[19] Jones, G.A. And Streit, M.: Galois groups, monodromy groups and cartographic groups. In Geometric Galois actions. 2. The inverse Galois problem, moduli spaces and mapping class groups, 25-65. London Math. Soc. Lecture Note Ser. 243, Cambridge University Press, 1997.

[20] Jones, G. A., Streit, M. And Wolfart, J.: Wilson's map operations on regular dessins and cyclotomic fields of definition. Proc. London Math. Soc. (3) 100 (2010), no. $2,510-532$.

[21] Kazaz, M.:, Finite groups and coverings of surfaces. PhD thesis, University of Southampton, 1997.

[22] Klein, F.: Über die Transformationen siebenter Ordnung der elliptischen Functionen. Math. Ann. 14 (1878/79), 428-497.

[23] Macbeath, A. M.: On a theorem of Hurwitz. Proc. Glasgow Math. Assoc. 5 (1961), 90-96. 
[24] Singerman, D.: Automorphisms of maps, permutation groups and Riemann surfaces. Bull. London Math. Soc. 8 (1976), 65-68.

[25] Singerman, D.: Finitely maximal Fuchsian groups. J. London Math. Soc. (2) 6 (1972), 29-38.

[26] Streit, M.: Field of definition and Galois orbits for the Macbeath-Hurwitz curves. Arch. Math. (Basel) 74 (2000), 342-349.

[27] Streit, M. and Wolfart, J.: Characters and Galois invariants of regular dessins. Rev. Mat. Complut. 13 (2000), 49-81.

[28] Streit, M. and Wolfart, J.: Cyclic projective planes and Wada dessins. Doc. Math. 6 (2001), 39-68.

[29] Takeuchi, K.: Commensurability classes of arithmetic triangle groups. J. Fac. Sci. Univ. Tokyo Sect. IA Math. 24 (1977), 201-212.

[30] Voevodsky, V.A. and Shabat, G.: Equilateral triangulations of Riemann surfaces, and curves over algebraic number fields. Soviet Math. Dokl. 39 (1989), 38-41.

[31] Walsh, T. R. S.: Hypermaps versus bipartite maps. J. Combin. Theory Ser. B 18 (1975), 155-163.

[32] Wilson, S. E.: Operators over regular maps. Pacific J. Math. 81 (1979), 559-568.

[33] Wolfart, J.: Triangle groups and Jacobians of CM type. Preprint available at www . math. uni-frankfurt.de/ wolfart/.

[34] Wolfart, J.: The "Obvious" part of Belyi's theorem and Riemann surfaces with many automorphisms. In Geometric Galois actions. 1. Around Grothendieck's esquisse d'un programme, 97-112. London Math. Soc. Lecture Note Ser. 242, Cambridge University Press, 1997.

[35] Wolfart, J.: ABC for polynomials, dessins d'enfants, and uniformization - a survey. In Elementare und Analytische Zahlentheorie (Tagungsband), 313-345. Proceedings ELAZ-Conference May 24-28, 2004.

(Also available at www.math.uni-frankfurt.de/ wolfart/.)

Received February 17, 2011.

Marston D. E. Conder: Department of Mathematics, University of Auckland, Private Bag 92019, Auckland, New Zealand.

E-mail: m.conder@auckland.ac.nz

Gareth A. Jones: School of Mathematics, University of Southampton, Southampton SO17 IBJ, UK.

E-mail: G.A.Jones@soton.ac.uk

Manfred Streit: Usinger Str. 56, D-61440 Oberursel, Germany.

E-mail: Manfred.Streit@bahn.de

Jürgen Wolfart: Mathematisches Seminar, Goethe Universität, Postfach 111932, D-60054 Frankfurt a.M., Germany.

E-mail: wolfart@math.uni-frankfurt.de

Marston Conder was supported by the Alexander von Humboldt Foundation. Jürgen Wolfart was supported by the Deutsche Forschungsgemeinschaft, project Wo199/4. 